\title{
Assessing the Quality of Knee Component Position Following Kinematically Aligned Total Knee Arthroplasty
}

\author{
Raj R. Thakrar and Sam Oussedik
}

\section{Key Points}

- Kinematically aligned (KA) knee components are implanted using the patient's joint surfaces and ligament tensions for reference rather than mechanical axes.

- This alternative alignment requires alternative assessment of post-operative radiographs.

- Implant positioning must be measured against the patient's own anatomy and as such is likely to result in valgus femoral positioning, varus tibial positioning and a resultant oblique joint line in the majority of patients.

- Evaluating three-dimensional images allows assessment in all three planes and will provide more accurate information regarding rotational alignment.

\subsection{Introduction}

Radiological assessment following knee arthroplasty surgery remains an essential aspect of routine post-operative care. Although outcomes after total knee arthroplasty (TKA) are increasingly focused on functional outcome scores, conventional radiographs still have a major role in the

R. R. Thakrar

Department of Orthopaedics, East and North Hertfordshire NHS Trust, Hertfordshire, UK

S. Oussedik $(\square)$

Department of Orthopaedics, University College

London Hospital NHS Trust, London, UK diagnosis and management of complications following surgery. In particular, component alignment in the coronal plane has been highlighted as playing an important role in implant survivorship of mechanically aligned (MA) TKA [1-4].

The Knee Society Total Knee Arthroplasty Roentgenograpic Evaluation and Scoring System published originally in $1989[5,6]$ is based on the anatomical axis, and allows for a systematic approach to the reporting of radiographs following TKA, providing a universally common method. However, the assessment and, more importantly, interpretation of post-operative TKA alignment may be influenced by the surgical philosophy adopted.

In this chapter, we aim to discuss how kinematic alignment (KA) in TKA may influence this interpretation of post-operative radiographs. Furthermore, we question whether conventional methods of short limb anteroposterior and lateral radiographs alone are sufficient in assessing the KA TKA.

\subsection{What Is Kinematic Alignment?}

Movement of the knee joint is achieved through the biomechanical interaction of the soft tissue component (ligaments and menisci) together with femoral and tibial articulating surfaces. Mean femoral joint angle (FJA) is approximately 
$3^{\circ}$ valgus and tibial joint angle (TJA) measures $3^{\circ}$ varus to their respective mechanical axes [7]. Consequently, mean constitutional knee joint alignment is $3^{\circ}$ varus to the mechanical axis of the lower limb.

First described by Freeman et al., the MA approach to TKA remains the gold standard. The technique aims to create a neutral lower limb alignment. This is achieved through preparing the distal femoral and proximal tibial cuts perpendicular to their respective mechanical axes. In addition, the posterior femoral condyle is cut in $3^{\circ}$ external rotation. The net effect is thought to be equal load distribution through a newly orientated joint line with favourable survivorship outcomes reported when this is achieved $[3,8,9]$.

More recently, however, work by Stephen Howell and colleagues has suggested an alternative approach to alignment in TKA, formally referred to as KA. Much of the work on KA has been driven by high patient dissatisfaction rates following the MA TKAs [10] bringing into question this surgical philosophy. KA works on the principle of correcting the arthritic deformity to restore the patient's own constitutional joint orientation, delivering a more "personalized" joint replacement. Considering the amount of bone and cartilage loss, resurfacing of the joint is achieved by adapting the bone resection thickness to match the implant thickness.

Appreciation of the KA approach requires an understanding of the kinematic axis of the knee joint. Much of the biomechanical rationale behind KA was introduced by Hollister et al. in the early 1990s [11] and more recently by the work of Eckhoff et al. [12]. Whilst the mechanical axis of the knee is based on a 2D schema of the joint (coronal and sagittal planes), the kinematic axis refers to its $3 \mathrm{D}$ orientation. By definition, the three kinematic axes include:

- Primary transverse axis (or cylindrical or transcondylar axis):

This passes through the centre of a circle fit to the articular surface of the medial and lateral femoral condyles and represents the axis about which the tibia flexes on the femur from $10^{\circ}$ to $120^{\circ}$.
- Secondary transverse axis:

This axis is parallel and proximal to the primary axis and is the transverse axis about which the patella flexes and extends on the femur.

- Longitudinal axis:

This is represented by the longitudinal axis of the tibia about which the tibia internally and externally rotates on the femur. The longitudinal axis is perpendicular to the primary and secondary axes.

The key operative goal of KA is to co-align the transverse axis of an appropriately sized femoral component to the primary transverse axis of the femur with the aim of restoring the normal interrelationships amongst the three axes.

\subsection{How Does Implant Position Vary Between MA and KA?}

To date, much of the literature evaluating postoperative alignment in TKA is based on the mechanically aligned knee using the aforementioned standardised radiographic views. The radiographic assessment of the MA knee will often demonstrate the joint to be in $4-6^{\circ}$ of valgus (tibiofemoral anatomic angle) with optimal range reported as $2-7^{\circ}$ by Fang et al. [8]. The alignment of the femoral component usually lies in $5-9^{\circ}$ of valgus relative to the long axis of the femur [13]. The tibial component is placed perpendicular to the long axis of the tibia. Ritter et al. reported in their series of 6070 TKAs with a minimum follow-up of 2 years that implant failure was most likely to occur if placed $<90^{\circ}$ relative to the tibial axis (i.e. valgus) and the femoral component $>8^{\circ}$ valgus.

In contrast, component alignment in a $\mathrm{KA}$ prosthetic knee is somewhat more variable. A study by Dossett et al. [14] reported their shortterm radiological results of an RCT comparing kinematically and mechanically aligned knee joints. They noted a tendency of placing the femoral component in a greater degree of valgus and tibial component in varus in the KA group. Importantly, they reported that the overall lower 
limb mechanical alignment was similar amongst the two groups with an average hip-knee-ankle angle of $0.3^{\circ}$ varus in the KA group compared to $0^{\circ}$ in the MA group. They concluded that the native joint line obliquity (best appreciated on weight-bearing long leg alignment views) was more closely restored in the KA group in comparison to the MA joints.

The results of this study have been echoed in a recent systematic review by Lee et al. [15] comparing KA and MA TKA. They concluded that whilst overall knee and limb alignments are similar amongst the two groups, the individual component alignment tends to be positioned in a greater degree valgus and varus in the femur and tibia, respectively, in the KA group. They also went on to conclude that the joint line obliquity in the KA group resembled that of the normal knee joint, something MA TKA fails to achieve.

\subsection{Approach to Radiological Assessment of the KA TKA}

Conventional weight-bearing anteroposterior and lateral radiographs are routinely used to assess the quality of implant positioning. Unlike in MA TKAs, with KA the ipsilateral pre-operative radiographs or the normal contralateral side knee is used as a reference for comparison.

Using these radiographs, component alignment in the coronal plane can be evaluated as the angle created between the anatomical axis of the bone and a line tangential to the articulating surface of the respective components with the aim of matching these angles pre- and postoperatively (Fig. 28.1). Furthermore, the coronal plane also allows assessment of the joint line obliquity (Fig. 28.2) on weight-bearing views.
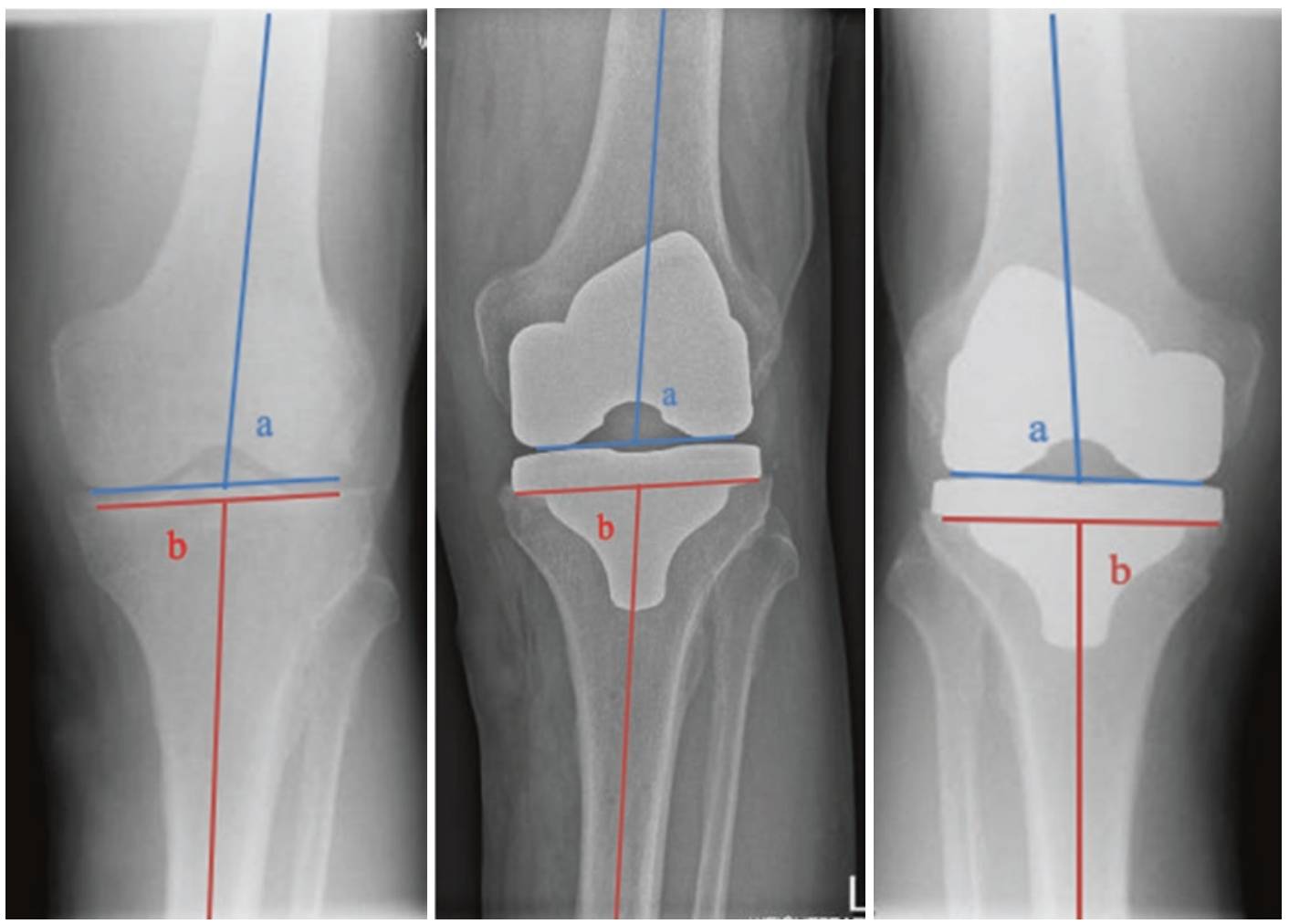

Fig. 28.1 Evaluation of component position using the anatomical axis. Comparison of pre-operative and postoperative angles demonstrates restoration of LDFA (a) and MPTA (b) angles following KA TKA (central image).
In contrast, a change in these angles is noted on the contralateral knee where MA approach was utilised (right image) 


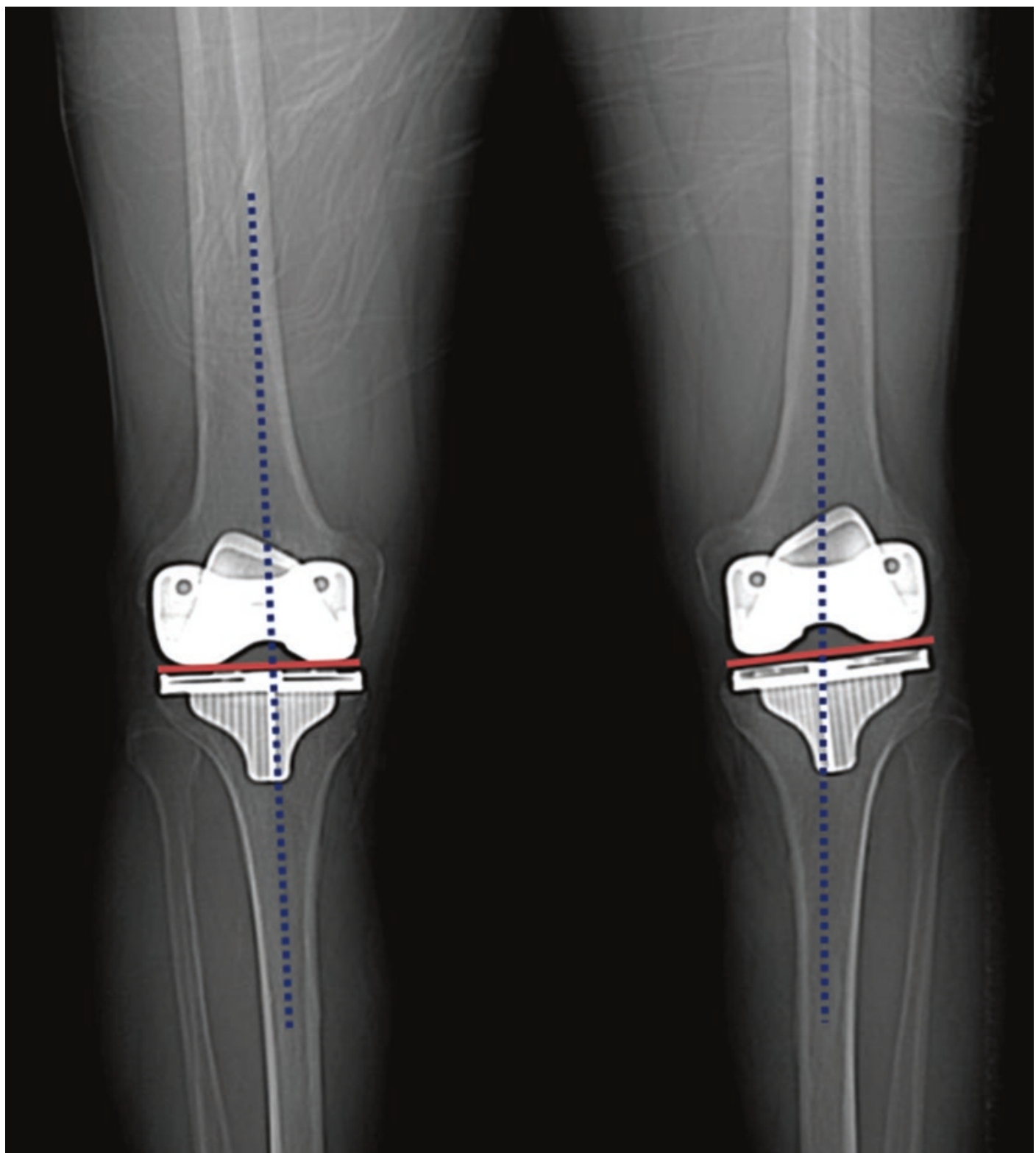

Fig. 28.2 Composite shows (1) the KA TKA (left knee) restores the natural joint line obliquity (red line) and maintains natural limb alignment (blue line) despite the

On lateral view, assessment of the femoral component offset gives an indication of whether the femoral component has been over-/undersized or excessively anteriorised. The posterior condylar offset is defined as the maximum thickness of the posterior condyle projected posteriorly to a line tangential to the posterior cortex of appearance of a varus tibial component anatomical alignment; (2) the MA TKA (right knee) changes the natural joint line obliquity (red line)

the femur (Fig. 28.3). Decreasing offset potentially restricts knee range of movement secondary to impingement of the tibial component on the posterior femoral shaft. Equally, excessive anteriorisation of the femoral component can lead to overstuffing of the patellofemoral compartment leading to poor outcomes. Assessment 

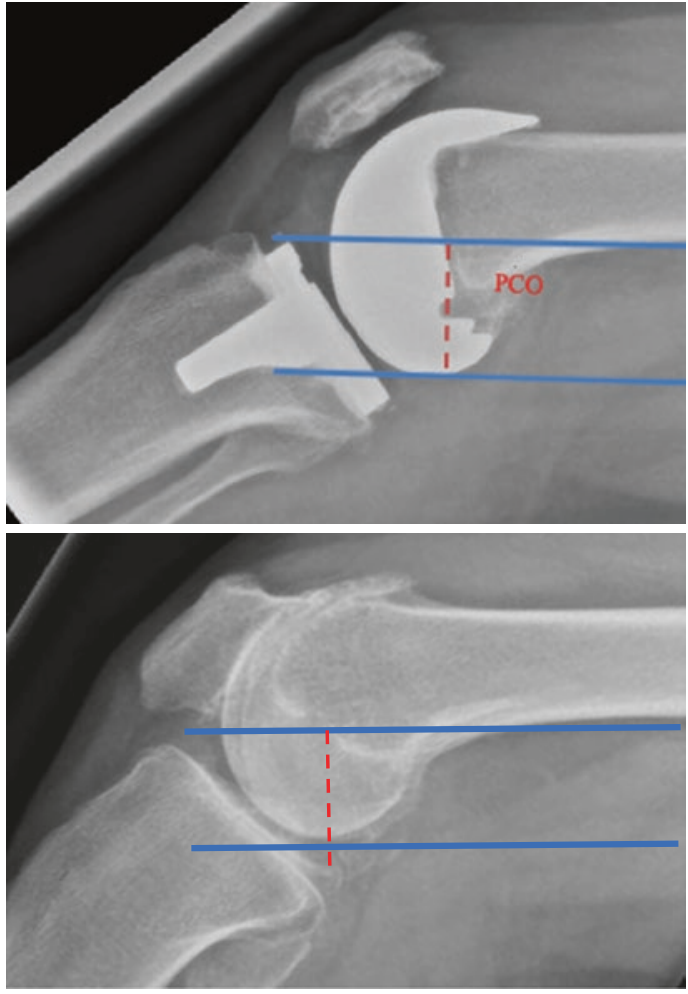

Fig. 28.3 Measurement of posterior condylar offset (PCO) pre- and post-op. Identified as the perpendicular distance between two parallel lines representing posterior cortex of femur and posterior femoral condyle

of the posterior condyle contour matching that of the femoral component is also a useful method of assessing femoral sizing in the sagittal plane.

The tibial component is typically positioned where it can provide maximal bone coverage whilst optimising patellar tracking. Restoration of tibial slope ensures joint stability in the AP plain whilst allowing for deep flexion of the knee and maintaining knee kinematics. The posterior tibial slope is obtained from the intersection of a line drawn through the mid-shaft of the tibia and a line tangential to the tibial component (Fig. 28.4).

Maintenance of joint line height also plays an important role in knee kinematics, influencing knee range of movement and patellar femoral joint contact forces [16]. This is likely through its effect on the functionality of the posterior cruciate ligament. Assessment of joint line height can be made on lateral view radiographs. Typically, it is measured as the perpendicular distance

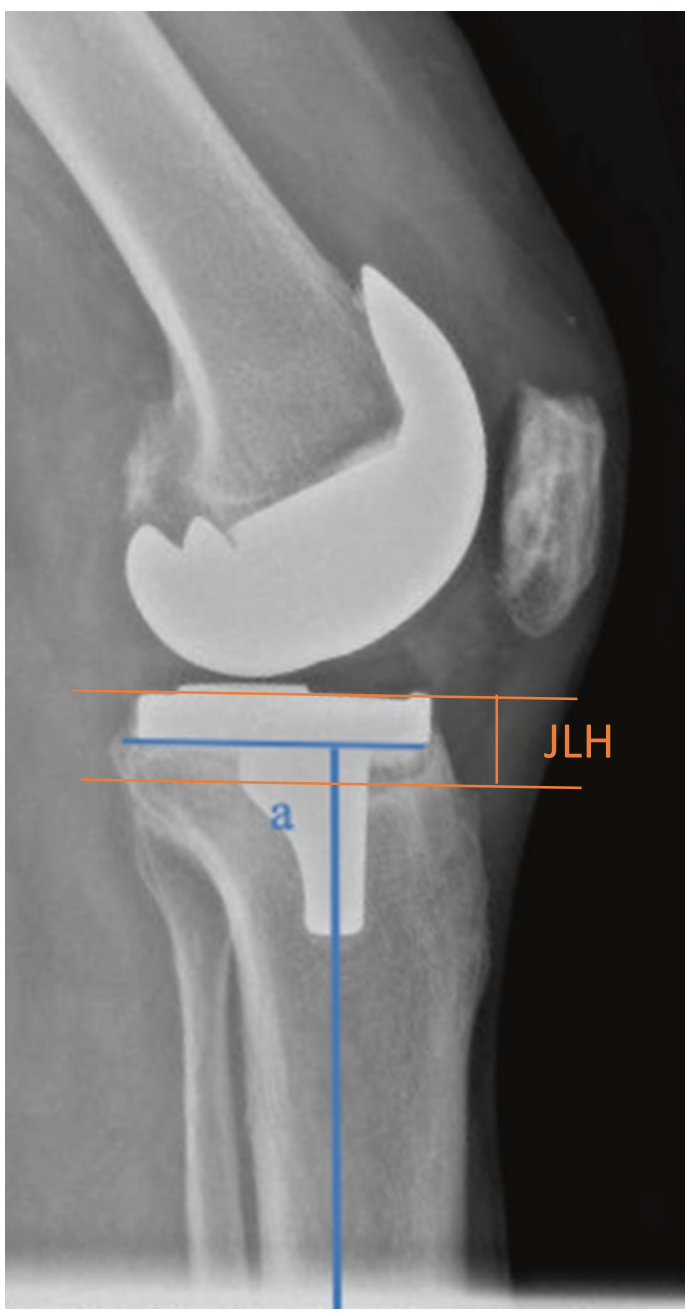

Fig. 28.4 Assessment of tibial slope (a) and joint line height (JLH)

between superior margin of the tibial tubercle and the weight-bearing parallel surface of the tibial component (Fig. 28.4).

Post-operative patella baja can negatively influence the outcomes for TKA through patellar maltracking and furthermore restricting range of movement. It is defined as a decrease in length of the patellar tendon by $10 \%$ of its preoperative value. Surgical techniques such as excessive Hoffa's fat pad excision resulting in tendon ischaemia are common causes for patella baja postTKA [17-19]. Equally, it is important to note that factors such as implant design and elevation of the joint line can also influence patellar height 
measurement with bi-cruciate-substituting knees demonstrating a more similar pattern of patellar tendon shortening during flexion to the native knee joint as compared with cruciate-retaining knee designs [20]. Whilst there are a number of recognised techniques for measurement of patellar height, the Insall-Salvati ratio has a number of theoretical advantages in that it is not influenced by the position of the joint line, size of the knee, position of the knee or radiographic magnification. The measurement was first described in 1971 [21]. It is calculated as the ratio of the length of the patellar tendon to the diagonal length of the patella (Fig. 28.5). A later modi-

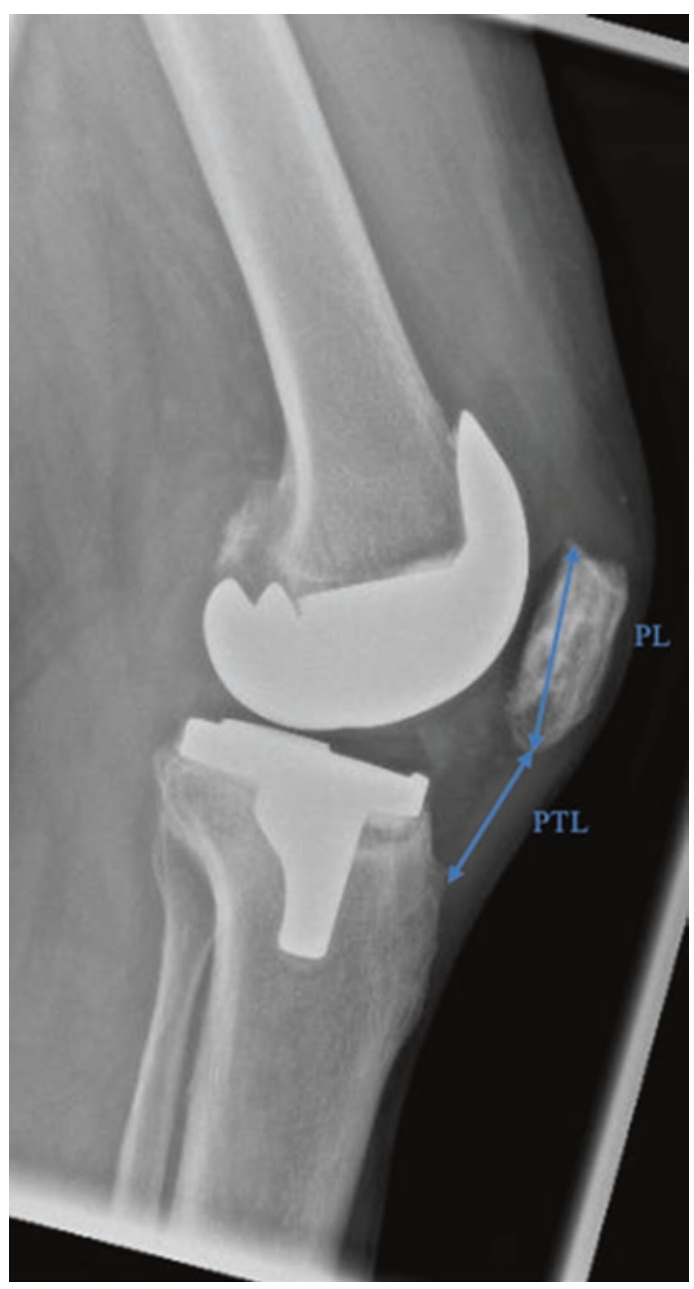

Fig. 28.5 Assessment of patellar height. Original description of the Insall-Salvati ratio expressed as a ratio of the patellar tendon length (PTL) over the patellar length (PL) fication of this measurement by Grelsamer and Meadows was introduced in 1992 to compensate for the ambiguity of identifying the true patella and patellar tendon lengths [22]. This modified value may be used as an adjunct to the InsallSalvati ratio.

\subsection{Is Traditional Short Leg Radiographic Assessment of Kinematic TKA Sufficient?}

It is traditional practise that assessment of component position and overall tibiofemoral alignment post-TKA be made on short leg radiographs. In the outpatient setting, these are easier to perform and limit the degree of radiation exposure to the patient.

Whilst there is sufficient evidence to support that this method delivers an adequate degree of clinical information [23], a number of more recent studies have questioned accuracy of short leg views when compared to hip-kneeankle standing long leg views for evaluation of coronal alignment [24, 25]. Furthermore, whilst non-weight-bearing radiographs provide information on component alignment relative to the anatomical axis of the femur and tibia, they fail to demonstrate exactly how the knee prosthesis is functionally loaded, assessment of which is relevant in both MA and KA philosophy. A study by Hutt et al. [26] highlighted the importance of this. They evaluated postop radiographs of $50 \mathrm{KA}$ TKAs. Their results reported that when looking at the tibial component relative to the mechanical axis of the tibia (as one would do on a short leg film assessment), a misleading, excessive degree of varus malalignment was noted $(66 \%$ outliers to the safe zone of $>3^{\circ}$ varus); however, when assessing the joint line angle on weight-bearing long leg views, this outlier group was significantly smaller (12\%). Hutt concluded that KA TKA often produces a joint line angle in varus relative to the mechanical axis of the tibia; when weight bearing, however, the actual joint line orientation becomes more acceptable, and this may explain promising early results in terms 
of survivorship of the KA knee joint despite $\mathrm{X}$-ray appearances.

Despite this, however, it is generally considered that weight-bearing long leg views are more relevant in the assessment of the MA TKA or in the research setting. For KA TKA, the traditional short leg views provide sufficient information to allow comparison of component position to preoperative distal femoral and proximal tibial alignment in both coronal and surgical planes as described earlier.

\subsection{Role of CT Evaluation}

Thus far, this chapter has described the approach to $2 \mathrm{D}$ coronal and sagittal plane assessment of implant position following KA TKA. Currently, there is no literature to support the superiority of a 3D imaging modality such as 3D computer tomography (3D-CT) over $2 \mathrm{D}$ radiographs in assessing the KA TKA. We attribute this to the fact that KA is a relatively new concept with much of the current literature being focused on the assessment of patient outcomes and implant survivorship. What is evident, however, is that whilst the standard views discussed thus far allow for varus/valgus and AP positioning of the components to be assessed, they are of limited value on assessment of axial rotational alignment [27-29]. Furthermore, a number of studies have identified challenges relating to the accurate assessment of subtle malposition on plain radiographs owing to variations in limb position and magnification [30, 31]. Early described methods of assessment of rotation are limited to implant design and $[32,33]$; hence, the current gold standard for assessment of rotation involves cross-sectional imaging in the form of CT scan [34], which is the case irrespective of whether an MA or KA philosophy has been adopted.

With regard to the KA knee, as discussed earlier, this philosophy is focused on a 3D model of the knee joint. In this scenario, CT may play a role in assessing, in particular, the position of the femoral component in the axial plane. A study by Hirschmann et al. compared the accuracy of conventional radiographs, transverse 2D-CT and 3D-CT reconstruction in assessing the position and orientation of TKA components. They concluded that their protocol of 3D-CT reconstruction for the assessment of rotational, sagittal and coronal orientation of the components reduced measurement errors as evident through less variability in inter- and intra-observer error. A limitation of CT, however, remains the inability to routinely perform weight-bearing imaging, which as mentioned earlier plays an important role when it comes to understanding functional loading of the joint. Hence, until such imaging becomes routinely available, it would have to be proposed that weight-bearing radiographs remain a useful alternative.

Equally, it is also important at this point to consider the clinical relevance of routine assessment of the axial orientation in KA TKA. Based on the surgical strategy of a measured resection tool adopted in this technique, the likelihood of failing to perform a cut parallel to the posterior femoral condyles is theoretically low. This, combined with a larger tolerance for axial orientation of the tibial component (in the order of $30-40^{\circ}$ ), may suggest that the overall axial orientation of the KA TKA (contrary to MA TKA), is unlikely to become a significant cause of clinical issues and therefore has little merit in being assessed.

\subsection{Summary}

KA TKA is becoming an increasingly popular philosophy with promising early reports on functional outcome and patient satisfaction. Assessment of the functionally loaded joint appears to be a key component in evaluation of the KA TKA. Conventional methods of short limb X-rays with anatomical assessment of component position provide sufficient information, especially when used in the context of referencing off pre-operative or contralateral normal limb radiographs. There is, however, some suggestion in the literature that short leg views may be misinterpreted as showing component malposition with excessive varus/valgus component alignment as compared to the acceptable safe 
zones when adopting the KA strategy. Equally the role of post-operative CT scan remains inconclusive in routine assessment of implant position.

Pearls and Pitfalls

1. KA in TKA has promising early clinical results.

2. Current approach to short leg views in postoperative assessment is adequate in assessing the KA joint.

3. Comparison of post-operative radiographs to either ipsilateral pre-operative or contralateral side normal radiographs is important as part of the evaluation process for assessment of accuracy of component positioning.

4. CT imaging in addition to long leg views plays a more important role in the evaluation of the MA TKA or in the context of research. Their role in routine assessment of the KA TKA remains inconclusive.

\section{Clinical Case}

A 62-year-old gentleman with bilateral knee osteoarthritis presented with severe mechanical pain in his left knee (Figs. 28.6 and 28.7). The limb was significantly varus aligned, but correctable. The patient was implanted with a KA medial pivot TKA design, using manual instrumentation and a callipered technique (Fig. 28.8). When planning and performing this case, the difficulty was in estimating the bone loss in the medial compartment. On comparison with the
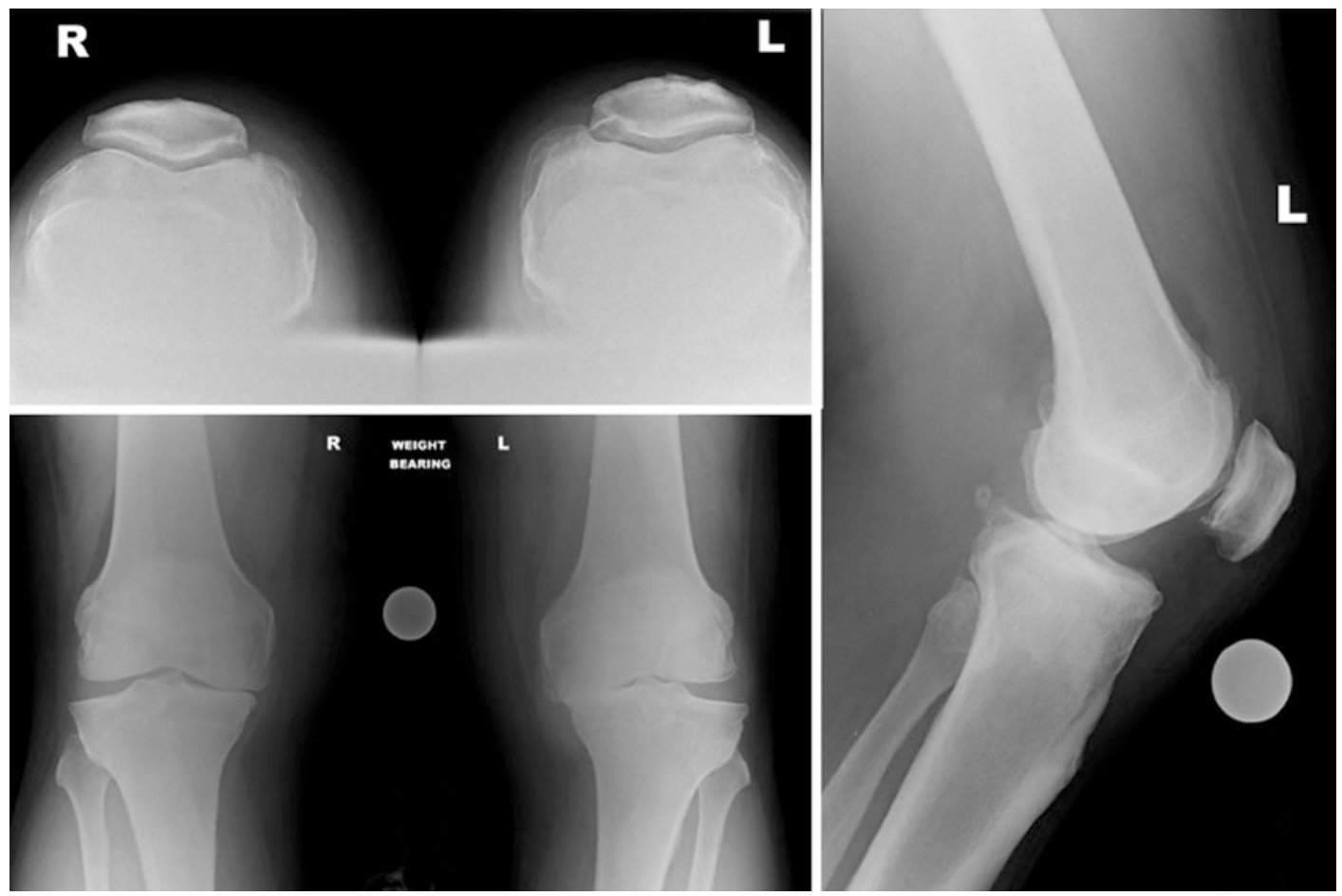

Fig. 28.6 Bilateral knee osteoarthritis primarily affecting the medial knee compartment, being more severe on the left knee 


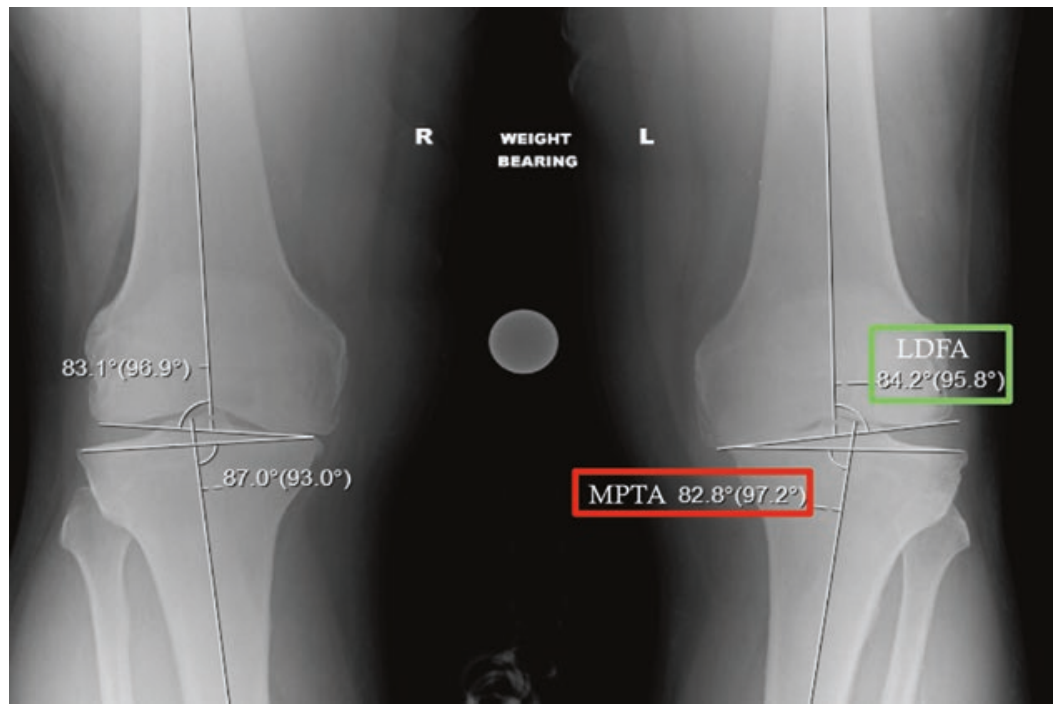

Fig. 28.7 Measurement of anatomical lateral distal femoral (LDFA) and medial proximal tibial (MPTA) angles on short film. The right knee is likely to have negligible bone loss and therefore may serve to estimate the prearthritic orientation of the femoral and tibial joint lines.
The bone loss was estimated to be $1 \mathrm{~mm}$ on the distal femoral condyle $\left(84.2^{\circ}-83.1^{\circ}=1.1^{\circ}\right)$ and $4 \mathrm{~mm}$ on the medial tibial plateau $\left(82.8^{\circ}-87^{\circ}=4.2^{\circ}\right)$, as $1^{\circ}$ of additional knee deformity approximately corresponds to $1 \mathrm{~mm}$ of articular surface bone loss right knee, which shows negligible bone loss, a $4 \mathrm{~mm}$ and a $1 \mathrm{~mm}$ loss of bone were estimated on the tibial and femoral sides, respectively.

The quality control for kinematic positioning of knee implants can be done intraoperatively, rather than on the post-operative radiograph alone. This is achieved through calliper measurement of the width of each bone cut, compensating for cartilage and bone loss, with the aim of matching the component thickness. As calliper measurements are precise, this method is indisputably the best way to guarantee correct kinematic positioning. Post-operatively, assessment of the quality of a kinematic implantation is possible by comparing radiographic orientation of artificial and native joint lines (distal femur and proximal tibia). For comparison, it is possible to use either the contralateral knee (ideally on post-operative frontal radiograph capturing both knees) or the preoperative images of the operated knee. In this specific case with significant bone loss, it is sensible to use the contralateral knee for the post-operative radiographic quality control (Figs. 28.7 and 28.9). While short films are sufficient for assessing the quality of a kinematic implantation, long films are also valuable and inform on the prosthetic limb alignment (hip-knee-ankle [HKA] angle).

While 2D images are subject to imprecisions in measurements (2D rendering of a $3 \mathrm{D}$ volume, high influence on frontal measures of knee rotation in axial and sagittal planes), 3D images enable more accurate assessment and should therefore become the standard. 

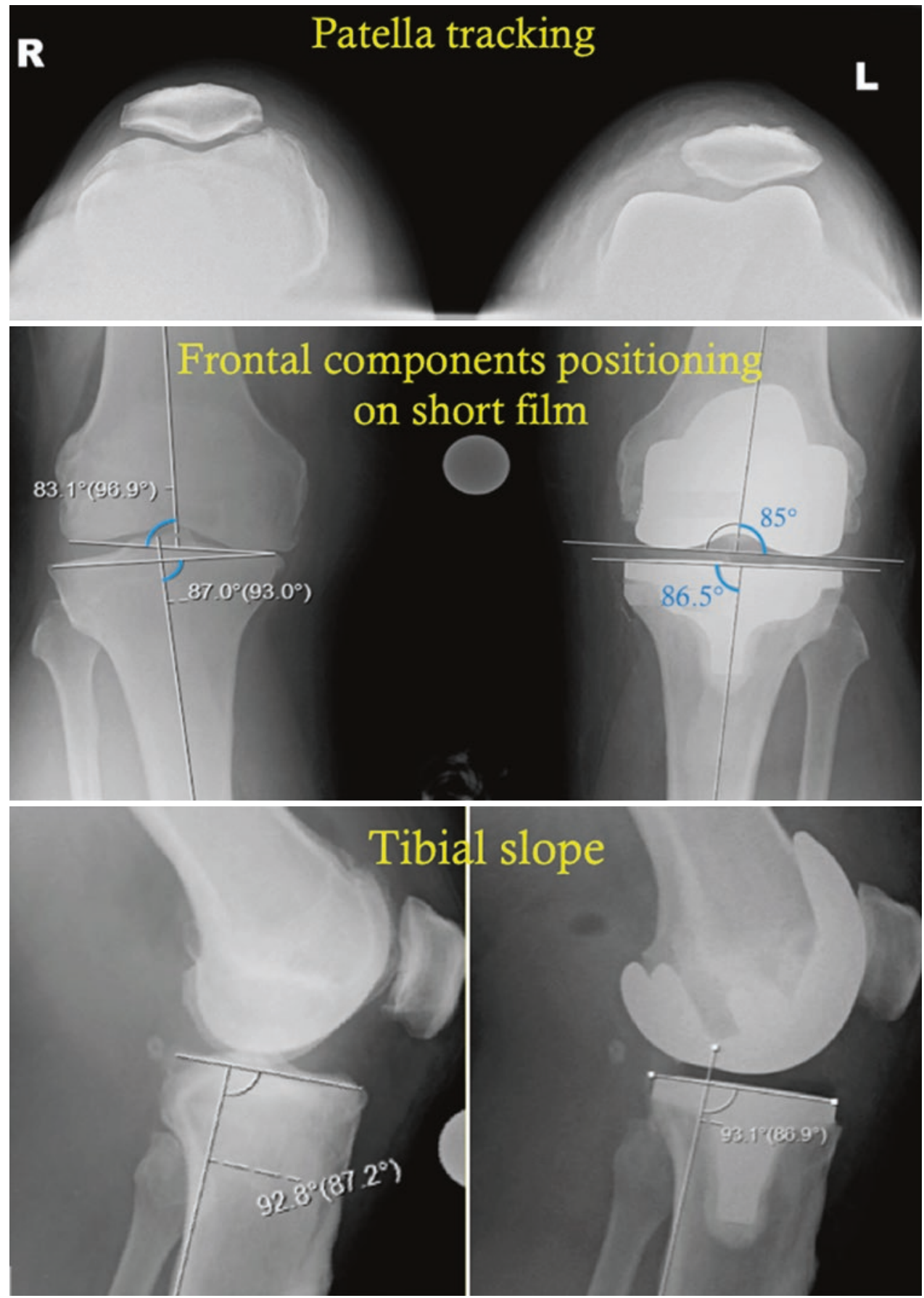

Fig. 28.8 Short film knee radiographs (skyline, frontal and lateral) enable assessment of the quality of a kinematic implantation by comparing the orientation of the joint lines between the right (native) and left (prosthetic) knees. The patellar tracking was considered excellent intraoperatively with the 'no thumb technique'; however, the patella was unexpectedly laterally shifted on the skyline view; its sig- nificance is unknown, and the patient had no complaints at her 1-year follow-up. Regarding frontal figures, it is likely that angular differences between knees are mainly the consequence of measurement imprecisions (2D radiograph), as the intraoperative calliper checks indicated accurate bone cuts, therefore guaranteeing correct restoration of the prearthritic distal femoral joint line orientation 


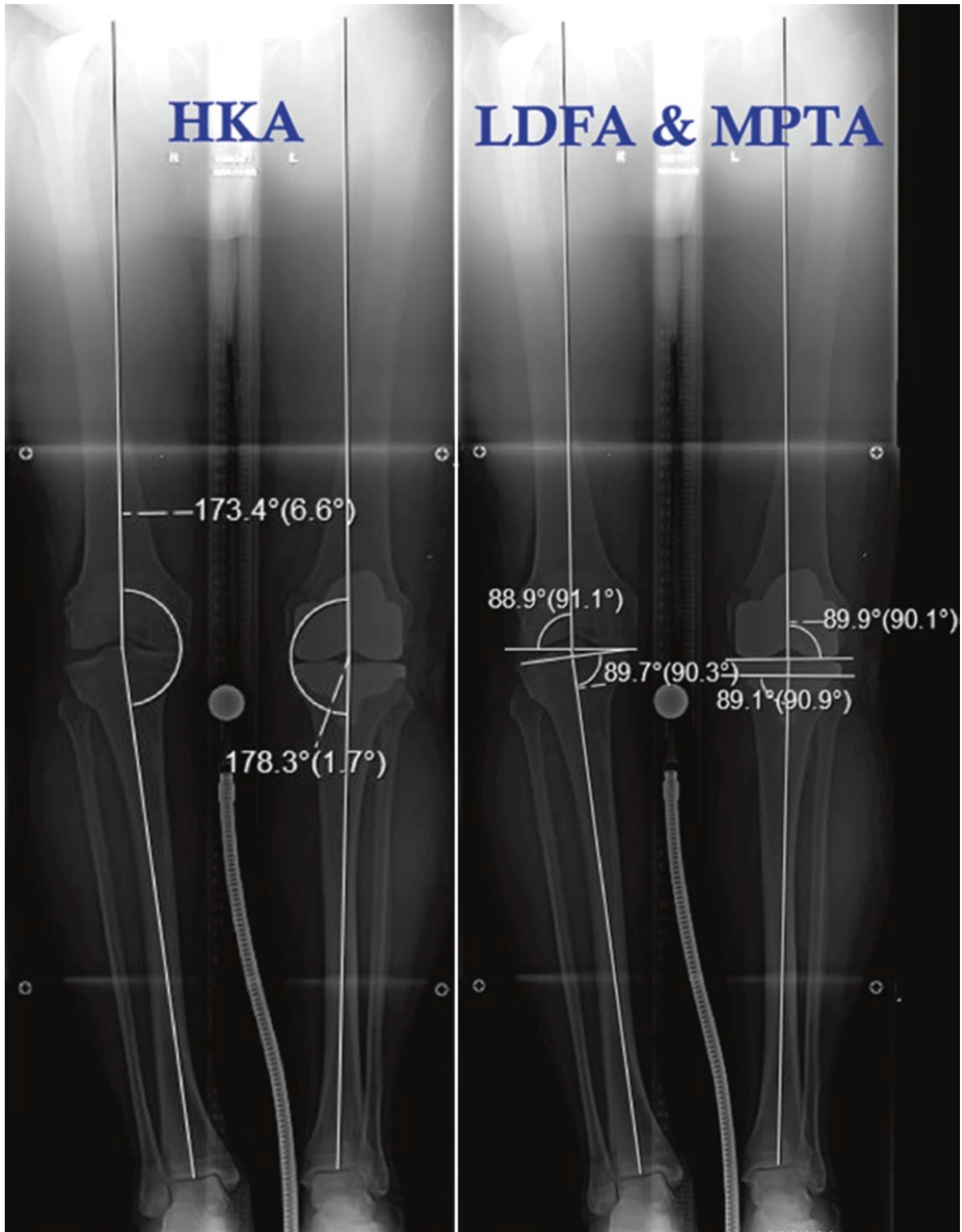

Fig. 28.9 Long films also enable assessment of the quality of the frontal component positioning (mechanical LDFA and MPTA angles), in addition to indicating the post-operative limb alignment by measurement of the hip-knee-ankle (HKA) angle. In this case, the frontal kinematic positioning of components resembles that of a mechanical implantation; nevertheless, the axial and sagittal positioning of components would still differ between techniques of alignment 


\section{References}

1. Longstaff LM, Sloan K, Stamp N, Scaddan M, Beaver R. Good alignment after total knee arthroplasty leads to faster rehabilitation and better function. J Arthroplast. 2009;24(4):570-8. [cited 2018 Jul 30]. http://www.ncbi.nlm.nih.gov/pubmed/18534396

2. Choong PF, Dowsey MM, Stoney JD. Does accurate anatomical alignment result in better function and quality of life? Comparing conventional and computer-assisted total knee arthroplasty. J Arthroplast. 2009;24(4):560-9. [cited 2018 Jul 30]. http://www.ncbi.nlm.nih.gov/pubmed/18534397

3. Ritter MA, Davis KE, Meding JB, Pierson JL, Berend ME, Malinzak RA. The effect of alignment and BMI on failure of total knee replacement. J Bone Joint Surg Am. 2011;93(17):1588-96. [cited 2018 Jul 30]. http:// www.ncbi.nlm.nih.gov/pubmed/21915573

4. Benjamin J. Component alignment in total knee arthroplasty. Instr Course Lect. 2006;55:405-12. [cited 2018 Jul 31] http://www.ncbi.nlm.nih.gov/ pubmed/16958475

5. Ewald FC. The Knee Society total knee arthroplasty roentgenographic evaluation and scoring system. Clin Orthop Relat Res. 1989;248:9-12. [cited 2018 Aug 12]. http://www.ncbi.nlm.nih.gov/pubmed/2805502

6. Scuderi GR, Bourne RB, Noble PC, Benjamin JB, Lonner JH, Scott WN. The new knee society knee scoring system. Clin Orthop Relat Res. 2012;470(1):3-19. https://doi.org/10.1007/s11999-011-2135-0. [cited 2018 Jul 30]. http://link.springer.com/

7. Moreland JR, Bassett LW, Hanker GJ. Radiographic analysis of the axial alignment of the lower extremity. J Bone Joint Surg Am. 1987;69(5):745-9. [cited 2018 Aug 11]. http://www.ncbi.nlm.nih.gov/ pubmed/3597474

8. Fang DM, Ritter MA, Davis KE. Coronal alignment in total knee arthroplasty. J Arthroplast. 2009;24(6):3943. [cited 2018 Aug 11]. http://www.ncbi.nlm.nih. gov/pubmed/19553073

9. Jeffery RS, Morris RW, Denham RA. Coronal alignment after total knee replacement. J Bone Joint Surg Br. 1991;73(5):709-14. [cited 2018 Aug 9]. http:// www.ncbi.nlm.nih.gov/pubmed/1894655

10. Bourne RB, Chesworth BM, Davis AM, Mahomed NN, Charron KDJ. Patient satisfaction after total knee arthroplasty: who is satisfied and who is not? Clin Orthop Relat Res. 2010;468(1):57-63. [cited 2018 Jun 3]. http://www.ncbi.nlm.nih.gov/pubmed/ 19844772

11. Hollister AM, Jatana S, Singh AK, Sullivan WW, Lupichuk AG. The axes of rotation of the knee. Clin Orthop Relat Res. 1993;290:259-68. [cited 2018 Jun 3]. http://www.ncbi.nlm.nih.gov/pubmed/8472457

12. Eckhoff DG. Three-dimensional mechanics, kinematics, and morphology of the knee viewed in virtual reality. J Bone Joint Surg. 2005;87(suppl_2):71. https://doi.org/10.2106/JBJS.E.00440. [cited 2018 Aug 12]. http://jbjs.org/cgi/doi/
13. Allen AM, Ward WG, Pope TL. Imaging of the total knee arthroplasty. Radiol Clin North Am. 1995;33(2):289-303. [cited 2018 Aug 12]. http:// www.ncbi.nlm.nih.gov/pubmed/7871170

14. Dossett HG, Swartz GJ, Estrada NA, LeFevre GW, Kwasman BG. Kinematically versus mechanically aligned total knee arthroplasty. Orthopedics. 2012;35(2):e160-9. [cited 2018 Aug 11]. http://www. ncbi.nlm.nih.gov/pubmed/22310400

15. Lee YS, Howell SM, Won Y-Y, Lee O-S, Lee SH, Vahedi $\mathrm{H}$, et al. Kinematic alignment is a possible alternative to mechanical alignment in total knee arthroplasty. Knee Surg Sports Traumatol Arthrosc. 2017;25(11):3467-79. [cited 2018 Sep 30]. http:// www.ncbi.nlm.nih.gov/pubmed/28439636

16. Figgie HE, Goldberg VM, Heiple KG, Moller HS, Gordon NH. The influence of tibial-patellofemoral location on function of the knee in patients with the posterior stabilized condylar knee prosthesis. J Bone Joint Surg Am. 1986;68(7):1035-40. [cited 2019 Mar 24]. http://www.ncbi.nlm.nih.gov/pubmed/3745240

17. Kayler DE, Lyttle D. Surgical interruption of patellar blood supply by total knee arthroplasty. Clin Orthop Relat Res. 1988;229:221-7. [cited 2019 Mar 24]. http://www.ncbi.nlm.nih.gov/pubmed/3349681

18. Weale AE, Murray DW, Newman JH, Ackroyd CE. The length of the patellar tendon after unicompartmental and total knee replacement. J Bone Joint Surg Br. 1999;81(5):790-5. [cited 2019 Mar 24]. http://www.ncbi.nlm.nih.gov/pubmed/10530838

19. Lee G-C, Cushner FD, Scuderi GR, Insall JN. Optimizing patellofemoral tracking during total knee arthroplasty. J Knee Surg. 2004;17(3):144-9; discussion 149-50. [cited 2019 Mar 24]. http://www. ncbi.nlm.nih.gov/pubmed/15366269

20. Brilhault J, Ries MD. Measuring patellar height using the lateral active flexion radiograph: effect of total knee implant design. Knee. 2010;17(2):148-51. [cited 2019 Mar 24]. http://www.ncbi.nlm.nih.gov/ pubmed/19720535

21. Insall J, Salvati E. Patella position in the normal knee joint. Radiology. 1971;101(1):101-4. [cited 2019 Mar 24]. http://www.ncbi.nlm.nih.gov/pubmed/5111961

22. Grelsamer RP, Meadows S. The modified InsallSalvati ratio for assessment of patellar height. Clin Orthop Relat Res. 1992;282:170-6. [cited 2019 Mar 24]. http://www.ncbi.nlm.nih.gov/pubmed/1516309

23. Patel DV, Ferris BD, Aichroth PM. Radiological study of alignment after total knee replacement. Int Orthop. 1991;15(3):209-10. https://doi.org/10.1007/ BF00192296. [cited 2018 Aug 12]. http://link. springer.com/

24. Park A, Stambough JB, Nunley RM, Barrack RL, Nam D. The inadequacy of short knee radiographs in evaluating coronal alignment after total knee arthroplasty. J Arthroplast. 2016;31(4):878-82. [cited 2018 Sep 12]. http://www.ncbi.nlm.nih.gov/pubmed/26410551

25. Abu-Rajab RB, Deakin AH, Kandasami M, McGlynn J, Picard F, Kinninmonth AWG. Hip-knee-ankle radiographs are more appropriate for assessment of 
post-operative mechanical alignment of total knee arthroplasties than standard AP knee radiographs. J Arthroplast. 2015;30(4):695-700. [cited 2018 Aug 12]. http://www.ncbi.nlm.nih.gov/pubmed/25702592

26. Hutt J, Massé V, Lavigne M, Vendittoli P-A. Functional joint line obliquity after kinematic total knee arthroplasty. Int Orthop. 2016;40(1):29-34. [cited 2018 Aug 12]. http://www.ncbi.nlm.nih.gov/pubmed/25795248

27. Dennis DA. Evaluation of painful total knee arthroplasty. J Arthroplasty. 2004;19(4 Suppl 1):35-40. [cited 2018 Sep 30]. http://www.ncbi.nlm.nih.gov/ pubmed/15190547

28. Mandalia V, Eyres K, Schranz P, Toms AD. Evaluation of patients with a painful total knee replacement. J Bone Joint Surg Br. 2008;90(3):265-71. [cited 2018 Sep 30]. http://www.ncbi.nlm.nih.gov/ pubmed/18310744

29. Toms AD, Mandalia V, Haigh R, Hopwood B. The management of patients with painful total knee replacement. J Bone Joint Surg Br. 2009;91(2):14350. https://doi.org/10.1302/0301-620X.91B2.20995. [cited 2018 Sep 30]. http://online.boneandjoint.org.uk.

30. Bäthis H, Perlick L, Tingart M, Lüring C, Zurakowski D, Grifka J. Alignment in total knee arthroplasty. A comparison of computer-assisted surgery with the conventional technique. J Bone Joint Surg Br. 2004;86(5):682-7. [cited 2018 Sep 30]. http://www. ncbi.nlm.nih.gov/pubmed/15274263

31. Skyttä ET, Lohman M, Tallroth K, Remes V. Comparison of standard anteroposterior knee and hip-to-ankle radiographs in determining the lower limb and implant alignment after total knee arthroplasty. Scand J Surg. 2009;98(4):250-3. [cited 2018 Sep 30]. http://www.ncbi.nlm.nih.gov/pubmed/20218424

32. Takai S, Yoshino N, Isshiki T, Hirasawa Y. Kneeling view: a new roentgenographic technique to assess rotational deformity and alignment of the distal femur. J Arthroplasty. 2003;18(4):478-83. [cited 2018 Aug 12]. http://www.ncbi.nlm.nih.gov/pubmed/12820092

33. Eckhoff DG, Piatt BE, Gnadinger CA, Blaschke RC. Assessing rotational alignment in total knee arthroplasty. Clin Orthop Relat Res. 1995;((318)):176-81. [cited 2018 Aug 12]. http://www.ncbi.nlm.nih.gov/ pubmed/7671514

34. Jazrawi LM, Birdzell L, Kummer FJ, Di Cesare PE. The accuracy of computed tomography for determining femoral and tibial total knee arthroplasty component rotation. J Arthroplast. 2000;15(6):761-6. [cited 2018 Aug 12]. http://www.ncbi.nlm.nih.gov/ pubmed/11021452

Open Access This chapter is licensed under the terms of the Creative Commons Attribution 4.0 International License (http://creativecommons.org/licenses/by/4.0/), which permits use, sharing, adaptation, distribution and reproduction in any medium or format, as long as you give appropriate credit to the original author(s) and the source, provide a link to the Creative Commons license and indicate if changes were made.

The images or other third party material in this chapter are included in the chapter's Creative Commons license, unless indicated otherwise in a credit line to the material. If material is not included in the chapter's Creative Commons license and your intended use is not permitted by statutory regulation or exceeds the permitted use, you will need to obtain permission directly from the copyright holder. 